\title{
Spherical Calogero model with oscillator/Coulomb potential: Classical case
}

\author{
Francisco Correa, ${ }^{1,2, *}$ Tigran Hakobyan, ${ }^{3,4, \dagger}$ Olaf Lechtenfeld, ${ }^{1, \$}$ and Armen Nersessian ${ }^{3,4, \S}$ \\ ${ }^{1}$ Leibniz Universität Hannover, Appelstraße 2, 30167 Hanover, Germany \\ ${ }^{2}$ Instituto de Ciencias Físicas y Matemáticas, Universidad Austral de Chile, Casilla 567, Valdivia, Chile \\ ${ }^{3}$ Yerevan State University, 1 Alex Manoogian Street, Yerevan 0025, Armenia \\ ${ }^{4}$ Tomsk Polytechnic University, Lenin Avenue 30, 634050 Tomsk, Russia
}

(Received 9 April 2016; published 7 June 2016)

\begin{abstract}
We construct the Hamiltonians and symmetry generators of Calogero-oscillator and Calogero-Coulomb models on the $\mathrm{N}$-dimensional sphere within the matrix-model reduction approach. Our method also produces the integrable Calogero-Coulomb-Stark model on the sphere and proves the integrability of the spin extensions of all these systems.
\end{abstract}

DOI: $10.1103 /$ PhysRevD.93.125008

\section{INTRODUCTION}

The rational Calogero model is one of the most important and most known integrable systems invented in the 20th century [1]. It describes $N$ particles on a line with a pairwise inverse-square interaction potential. Adding an external oscillator potential preserves its integrability $[2,3]$. Moreover, this model, as well as its generalizations associated with arbitrary root systems [4], were found to be superintegrable (i.e. they possess $2 N-1$ functionally independent constants of motion) [5]. The hyperbolic version of the Calogero model remains superintegrable [6], while its trigonometric counterpart inherits just the integrability [7]. These describe $N$ interacting particles on a circle or hyperbola, correspondingly. Attempts to construct integrable analogs of such systems in higher dimensions have been unsuccessful; see e.g. Ref. [8]. On the other hand, all these models can be interpreted as a single particle in $N$-dimensional Euclidean space subject to very particular nonisotropic potentials. From this viewpoint it is natural to modify their $r^{-2}$ radial dependence by putting this particle on an $N$-sphere or $N$-hyperboloid. This deformation retains the superintegrability but loses the multiparticle interpretation.

In our recent paper [9], we have indicated that the spherical or hyperbolic extensions of the rational Calogero potential associated with an arbitrary Coxeter group is the only possible superintegrable deformation of the $N$-dimensional oscillator and Coulomb systems. We also revealed explicit expressions for the constants of motion of the Calogero-Coulomb problem on Euclidean space in both the classical [9] and the quantum [10] cases. They involve an analog of the Runge-Lenz vector and its related algebra. From the other side, the hidden symmetries of the rational

\footnotetext{
*francisco.correa@itp.uni-hannover.de tigran.hakobyan@ysu.am *lechtenf@itp.uni-hannover.de §arnerses@ysu.am
}

Calogero-oscillator problem have been known for decades and are well investigated [4,11]. The integrable two-center counterpart of the Calogero-Coulomb system and an integrable Calogero-Coulomb-Stark system and their symmetry generators have been constructed as well [12].

However, it seems that the Calogero-oscillator and Calogero-Coulomb systems extended to the $N$-sphere or $N$-hyperboloid have not yet been investigated properly. This is the subject of the current paper. For arbitrary (positive) Coxeter root systems $\Delta_{+}=\{\alpha\}$, their Hamiltonians read

$$
\mathcal{H}=\frac{\boldsymbol{p}^{2}}{2} \mp \frac{(\boldsymbol{x} \cdot \boldsymbol{p})^{2}}{2 r_{0}^{2}}+\sum_{\alpha \in \Delta_{+}} \frac{g_{\alpha}^{2}(\alpha \cdot \alpha)}{2(\alpha \cdot \boldsymbol{x})^{2}}+V(\boldsymbol{x})
$$

with

$V_{\omega}=\frac{\omega^{2} r_{0}^{2}}{2} \frac{x^{2}}{x_{0}^{2}}, \quad V_{\gamma}=-\frac{\gamma}{r_{0}} \frac{x_{0}}{|\boldsymbol{x}|} \quad$ where $x_{0}^{2} \pm \boldsymbol{x}^{2}=r_{0}^{2}$

Here, the upper/lower sign corresponds to the sphere/ hyperboloid, $\boldsymbol{x}$ and $x_{0}$ are Cartesian coordinates in the ambient $(N+1)$-dimensional space, and $r_{0}$ is the radius of the $N$-dimensional sphere/hyperboloid. The vectors $\alpha$ from the set $\Delta_{+}$of positive roots uniquely characterize the Coxeter reflections, and the coupling constants $g_{\alpha}$ form a reflection-invariant discrete function. The original Calogero potential corresponds to the $A_{N-1}$ Coxeter system with the positive roots given in terms of the standard basis by $\alpha_{i j}=e_{i}-e_{j}$ for $i<j$. The reflections become the coordinate permutations in this particular case.

In the absence of the Calogero interaction $\left(g_{\alpha}=0\right)$, such systems are reduced to the spherical and pseudospherical Coulomb and oscillator systems, introduced a long time ago by Schrödinger and Higgs $[13,14]$. These systems have direct analogs of the hidden-symmetry generators of their Euclidean counterparts. However, their symmetry algebras 
are essentially nonlinear even when restricted to a constant energy surface. In the absence of the Calogero and oscillator/Coulomb potentials, the above Hamiltonian describes a free particle on the $\mathrm{N}$-dimensional sphere/ hyperboloid. Hence, the system (1.1) with vanishing potential $V(\boldsymbol{x})$ is nothing but the spherical/hyperbolic Calogero model associated with an arbitrary reflection group. Particle motion near the horizon of an extremal $(2 N+1)$-dimensional Perry-Myers black hole is an example of such a system [15].

We remark that the so-defined spherical Calogero model differs from the angular Calogero model investigated recently in Refs. [16-19]. More precisely, the conventional Calogero model on the $N$-dimensional sphere is given by the Hamiltonian

$$
\mathcal{H}_{0}=\frac{\boldsymbol{p}^{2}}{2}-\frac{(\boldsymbol{x} \cdot \boldsymbol{p})^{2}}{2 r_{0}^{2}}+\sum_{i<j} \frac{g^{2}}{\left(x_{i}-x_{j}\right)^{2}},
$$

while the angular Calogero Hamiltonian $\mathcal{H}_{\Omega}$ corresponding to the angular part of the Calogero system on $(N+1)$ dimensional flat space is defined by

$$
\begin{aligned}
r_{0}^{-2} \mathcal{H}_{\Omega}= & \frac{\boldsymbol{p}^{2}}{2}-\frac{(\boldsymbol{x} \cdot \boldsymbol{p})^{2}}{2 r_{0}^{2}}+\sum_{i<j} \frac{g^{2}}{\left(x_{i}-x_{j}\right)^{2}}+\sum_{i=1}^{N} \frac{g^{2}}{\left(x_{i}-x_{0}\right)^{2}} \\
& \text { with } \quad x_{0}=\sqrt{r_{0}^{2}-\boldsymbol{x}^{2}} .
\end{aligned}
$$

The goal of the current paper is to investigate the symmetries of the Calogero-oscillator and CalogeroCoulomb systems on the $\mathrm{N}$-dimensional sphere using the matrix-model reduction. For this purpose, first we reformulate the $N^{2}$-dimensional spherical oscillator/Coulomb system on the space of Hermitian $N \times N$ matrices. Then, reducing this system by the $\mathrm{SU}(N)$ adjoint action and fixing specific values of its generators in a standard way, we arrive at the desired Calogero-oscillator and CalogeroCoulomb systems (1.1). The $\mathrm{SU}(N)$ invariant polynomials in the matrix-model symmetry generators yield the correct integrals of motion for the reduced system. In a similar way, we find an integrable Calogero-Coulomb-Stark system on the sphere. We restrict ourselves to the spherical $A_{N-1}$ Calogero model (1.3) supplemented by the potentials (1.2). We do not consider the hyperboloid case, since the transition from the spherical one is straightforward.

The paper is organized as follows: In Sec. II, we present general properties of the classical oscillator and Coulomb systems on the sphere in a parametrization relevant for our purposes. In Sec. III, we construct the symmetry generators of the classical Calogero-oscillator and Calogero-Coulomb systems on the $N$-dimensional sphere using the matrixmodel reduction. In Sec. IV, we present the CalogeroCoulomb-Stark system and briefly discuss the spin generalizations of considered models.

\section{PRELIMINARY: OSCILLATOR AND COULOMB SYSTEMS ON THE SPHERE}

Let us briefly describe, following Ref. [14], the Coulomb and oscillator systems on an $N$-dimensional sphere of radius $r_{0}$, in parametrized $N$ Cartesian coordinates of the ambient $(N+1)$-dimensional space. In this parametrization, the sphere metric can immediately be obtained by the restriction of the flat metric on $\mathbb{R}^{N+1}$ :

$d s^{2}=h_{i j} d x_{i} d x_{j}=d \boldsymbol{x}^{2}+\left.d x_{0}^{2}\right|_{x_{0}^{2}+\boldsymbol{x}^{2}=r_{0}^{2}}=d \boldsymbol{x}^{2}+\frac{(\boldsymbol{x} \cdot d \boldsymbol{x})^{2}}{r_{0}^{2}-\boldsymbol{x}^{2}}$.

The phase space of the systems on the sphere is given by its cotangent bundle equipped with the canonical symplectic structure $d \boldsymbol{p} \wedge d \boldsymbol{x}$ and the canonical Poisson brackets $\left\{p_{i}, x_{j}\right\}=\delta_{i j}$. In these terms, the $\mathrm{SO}(N+1)$ isometries of the sphere are given by the generators $\left(L_{\mu \nu}\right)=\left(L_{0 i}, L_{i j}\right)$ via

$$
\begin{aligned}
L_{0 i} & =x_{0} p_{i}, \quad L_{i j}=x_{i} p_{j}-x_{j} p_{i}, \quad \text { and } \\
\left\{L_{\mu \nu}, L_{\rho \lambda}\right\} & =\delta_{\mu \lambda} L_{\nu \rho}+\delta_{\nu \rho} L_{\mu \lambda}-\delta_{\mu \rho} L_{\nu \lambda}-\delta_{\nu \lambda} L_{\mu \rho},
\end{aligned}
$$

where $x_{0}=\sqrt{r_{0}^{2}-x^{2}}$. The above $s o(N+1)$ algebra decomposes as

$$
\begin{array}{r}
\left\{L_{0 i}, L_{0 j}\right\}=L_{i j}, \quad\left\{L_{0 i}, L_{k j}\right\}=\delta_{i k} L_{0 j}-\delta_{i j} L_{0 k}, \\
\left\{L_{i j}, L_{k l}\right\}=\delta_{i l} L_{j k}+\delta_{j k} L_{i l}-\delta_{i k} L_{j l}-\delta_{j l} L_{i k},
\end{array}
$$

where the $L_{i j}$ 's generate the $s o(N)$ subalgebra.

The oscillator on the $N$-dimensional sphere is defined by the Hamiltonian [14]

$$
H_{\omega}=\frac{\boldsymbol{p}^{2}}{2}-\frac{(\boldsymbol{x} \cdot \boldsymbol{p})^{2}}{2 r_{0}^{2}}+\frac{\omega^{2} r_{0}^{2}}{2} \frac{\boldsymbol{x}^{2}}{x_{0}^{2}} .
$$

The symmetries of this Hamiltonian are given by the generators of the $\mathrm{SO}(N)$ angular momentum $L_{i j}$ defined in (2.2) and the hidden-symmetry generators

$$
I_{i j}=\frac{x_{0}^{2}}{r_{0}^{2}} p_{i} p_{j}+\frac{\omega^{2} r_{0}^{2}}{x_{0}^{2}} x_{j} x_{j}
$$

Note that these expressions can be obtained from those of the flat oscillator by the replacement

$$
x_{i} \rightarrow \frac{r_{0}}{x_{0}} x_{i}, \quad p_{i} \rightarrow \frac{L_{0 i}}{r_{0}}=\frac{x_{0}}{r_{0}} p_{i} .
$$

The symmetry algebra of the spherical system is essentially nonlinear [14]: 


$$
\begin{array}{r}
\left\{L_{i j}, I_{k l}\right\}=\delta_{j k} I_{i l}-\delta_{i l} I_{j k}-\delta_{i k} I_{j l}+\delta_{j l} I_{i k}, \\
\left\{I_{i j}, I_{k l}\right\}=-\omega^{2}\left(\delta_{i l} L_{j k}+\delta_{j k} L_{i l}+\delta_{i k} L_{j l}+\delta_{j l} L_{i k}\right) \\
-\frac{1}{r_{0}^{2}}\left(I_{i l} L_{j k}+I_{j k} L_{i l}+I_{i k} L_{j l}+I_{j l} L_{i k}\right) .
\end{array}
$$

The Hamiltonian can be expressed in terms of the symmetry generators,

$$
H_{\omega}=\frac{1}{2} \sum_{i} I_{i i}+\frac{\boldsymbol{L}^{2}}{2 r_{0}^{2}}, \quad \text { where } \boldsymbol{L}^{2}=\sum_{i<j} L_{i j}^{2} .
$$

In the $r_{0} \rightarrow \infty$ limit, the generators $L_{i j}$ and $I_{i j}$ reduce, respectively, to the angular momentum and Fradkin tensors. Together they form the $\mathrm{SU}(N)$ Poisson bracket algebra, which describes the symmetries of the standard $N$-dimensional oscillator [20].

The Coulomb system on the $N$-dimensional sphere possesses a similar structure [14]. It is defined by the Hamiltonian

$$
H_{\gamma}=\frac{\boldsymbol{p}^{2}}{2}-\frac{(\boldsymbol{x} \cdot \boldsymbol{p})^{2}}{2 r_{0}^{2}}-\frac{\gamma}{r_{0}} \frac{x_{0}}{x} \quad \text { with } \quad x=\sqrt{\boldsymbol{x}^{2}} .
$$

Its symmetry involves the $\mathrm{SO}(N)$ angular momentum tensor $L_{i j}$ and the analog of the Runge-Lenz vector,

$$
A_{i}=\frac{x_{0}}{r_{0}} \sum_{j} L_{i j} p_{j}-\frac{\gamma x_{i}}{x} .
$$

The square of the latter involves the energy,

$$
\boldsymbol{A}^{2}=\left(2 H_{\gamma}-\frac{\boldsymbol{L}^{2}}{r_{0}^{2}}\right) \boldsymbol{L}^{2}+\gamma^{2} .
$$

It is easy to see that the expression for the Runge-Lenz vector on the sphere can again be obtained from the one on flat space by the replacement (2.7). The symmetry algebra is given by the relations (2.4) and by

$$
\begin{aligned}
\left\{L_{i j}, A_{k}\right\} & =-\delta_{i k} A_{j}+\delta_{j k} A_{i}, \\
\left\{A_{i}, A_{j}\right\} & =2\left(H_{\gamma}-\frac{\boldsymbol{L}^{2}}{r_{0}^{2}}\right) L_{i j} .
\end{aligned}
$$

In the flat-space limit and on a fixed energy level, it is reduced to the $\mathrm{SO}(N, 1)$ or $\mathrm{SO}(N+1)$ symmetry for positive or negative energy, correspondingly.

\section{SPHERICAL CALOGERO-COULOMB AND CALOGERO-OSCILLATOR FROM MATRIX MODELS}

In this section, we construct the spherical Calogerooscillator and Calogero-Coulomb systems using the matrix-model reduction. More precisely, we consider the usual spherical oscillator and Coulomb systems on the space of $N \times N$ Hermitian matrices and then reduce them by the adjoint $\mathrm{SU}(N)$ action. As a result, we get the spherical Calogero-oscillator and Calogero-Coulomb systems suggested in Ref. [9]. This approach allows us to find explicit expressions for all symmetry generators (including the hidden ones). In addition, it is immediately generalized to superintegrable spin extensions of these models, as well as to an integrable spherical Calogero-CoulombStark model.

\section{A. Calogero-oscillator on the sphere}

Let us define the Hermitian matrix model for the oscillator on the sphere by the Hamiltonian

$\mathcal{H}_{\omega}^{\text {mat }}=\frac{1}{2} \operatorname{tr} \mathbf{P}^{2}-\frac{1}{2 r_{0}^{2}}(\operatorname{tr} \mathbf{P X})^{2}+\frac{\omega^{2} r_{0}^{2}}{2} \frac{\operatorname{tr} \mathbf{X}^{2}}{r_{0}^{2}-\operatorname{tr} \mathbf{X}^{2}}$.

Here $\mathbf{P}$ and $\mathbf{X}$ are Hermitian matrices containing, respectively, $N^{2}$ momenta and coordinates:

$$
\mathbf{P}=\sum_{a=1}^{N^{2}} P_{a} \mathbf{T}_{a}, \quad \mathbf{X}=\sum_{a=1}^{N^{2}} X_{a} \mathbf{T}_{a} .
$$

We have introduced a basis of orthonormalized $\mathrm{U}(N)$ generators,

$$
\left[\mathbf{T}_{a}, \mathbf{T}_{b}\right]=\imath \sum_{a} f_{a b c} \mathbf{T}_{c}, \quad \operatorname{tr} \mathbf{T}_{a} \mathbf{T}_{b}=\delta_{a b}
$$

Their explicit form can be set, in particular, by choosing $N$ matrices to be diagonal,

$$
\mathbf{T}_{(i-1) N+i}=\mathbf{E}_{i, i}, \quad 1 \leq i \leq N,
$$

where $\mathbf{E}_{i, j}$ has vanishing entries except for one in the $i$ th row and $j$ th column:

$$
\left[\mathbf{E}_{i, j}\right]_{i^{\prime} j^{\prime}}=\delta_{i i^{\prime}} \delta_{j j^{\prime}}
$$

The remaining $\mathbf{T}_{a}$ 's are selected from the following set of the $N(N-1)$ off-diagonal matrices:

$$
\begin{aligned}
\mathbf{T}_{(j-1) N+i} & =\frac{1}{\sqrt{2}}\left(\mathbf{E}_{j, i}+\mathbf{E}_{i, j}\right), \\
\mathbf{T}_{(i-1) N+j} & =\frac{l}{\sqrt{2}}\left(\mathbf{E}_{j, i}-\mathbf{E}_{i, j}\right), \quad 1 \leq j<i \leq N .
\end{aligned}
$$

In terms of the phase-space variables $\left(P_{a}, X_{a}\right)$, the equivalence of the matrix model (3.1) to the Hamiltonian of the $N^{2}$-dimensional spherical oscillator (2.5) becomes transparent: 
$H_{\omega}^{\mathrm{mat}}=\frac{1}{2} \sum_{a} P_{a}^{2}-\frac{1}{2 r_{0}^{2}}\left(\sum_{a} X_{a} P_{a}\right)^{2}+\frac{r_{0}^{2}}{x_{0}^{2}} \frac{\omega^{2} x^{2}}{2}$,

with $x$ and $x_{0}$ defined by

$$
x^{2}=\sum_{a=1}^{N^{2}} X_{a}^{2}, \quad x_{0}=\sqrt{r_{0}^{2}-x^{2}} .
$$

According to the previous section, the above system remains invariant under the action of the angular momentum and hidden symmetry generators

$L_{a b}=X_{a} P_{b}-X_{b} P_{a}, \quad I_{a b}=\frac{x_{0}^{2}}{r_{0}^{2}} P_{a} P_{b}+\frac{\omega^{2} r_{0}^{2}}{x_{0}^{2}} X_{a} X_{b}$.

These constants of motion obey Poisson bracket relations similar to (2.4), (2.8) and (2.9). They can be presented in matrix form:

$$
\begin{gathered}
\mathbf{L}=\sum_{a, b} L_{a b} \mathbf{T}_{a} \otimes \mathbf{T}_{b}=\mathbf{X} \wedge \mathbf{P}, \\
\mathbf{I}=\sum_{a, b} I_{a b} \mathbf{T}_{a} \otimes \mathbf{T}_{b}=\frac{x_{0}^{2}}{r_{0}^{2}} \mathbf{P} \otimes \mathbf{P}+\frac{\omega^{2} r_{0}^{2}}{x_{0}^{2}} \mathbf{X} \otimes \mathbf{X} .
\end{gathered}
$$

The matrix Hamiltonian (3.1) remains invariant under the adjoint $\mathrm{SU}(N)$ action:

$$
\mathbf{P} \rightarrow \mathbf{U P U}^{+}, \quad \mathbf{X} \rightarrow \mathbf{U X \mathbf { U } ^ { + }} .
$$

The related Noether current is given by a traceless Hermitian matrix with $s u(N)$-valued entries,

$$
\begin{aligned}
\mathbf{J} & ={ }_{l}[\mathbf{X}, \mathbf{P}], \quad J_{a}=\operatorname{tr} \mathbf{J} \mathbf{T}_{a}=-\frac{1}{2} \sum_{b, c} f_{a b c} L_{b c}, \\
\left\{J_{a}, J_{b}\right\} & =\sum_{c} f_{a b c} J_{c} .
\end{aligned}
$$

To perform the reduction, we diagonalize the coordinate matrix by the use of an $\mathrm{SU}(N)$ transformation (3.12). Then we fix the level surface $\mathbf{J}=$ const and take into account that the diagonalization of the coordinate matrix leads to the vanishing of the diagonal entries of $\mathbf{J}$. The following level surface reproduces the Calogero potential:

$$
J_{i j}=g\left(\delta_{i j}-1\right)
$$

As a result, the phase-space variables are mapped to $[1,4,21]$

$$
X_{i j}=x_{i} \delta_{i j} \quad \text { and } \quad P_{i j}=p_{i} \delta_{i j}+\imath g \frac{1-\delta_{i j}}{x_{i}-x_{j}} .
$$

Their diagonal entries define the coordinates and momenta of the $N$-dimensional Calogero system. Only these preserve the canonical Poisson bracket relations. Nevertheless, we keep the same notation for the reduced matrices (3.15). Note that the reduced momentum matrix $P_{i j}$ corresponds to the Lax matrix of the standard Calogero system [3].

Using the expressions for the $\mathrm{U}(N)$ generators (3.4) and (3.6), we get an explicit form of the orthogonal coordinates (3.2) under the reduction:

$$
\begin{aligned}
& X_{(j-1) N+i}= \begin{cases}x_{i} & \text { for } i=j, \\
0 & \text { for } i \neq j,\end{cases} \\
& P_{(j-1) N+i}= \begin{cases}p_{i} & \text { for } i=j, \\
\frac{\sqrt{2} g}{x_{i}-x_{j}} & \text { for } i<j, \\
0 & \text { for } i>j .\end{cases}
\end{aligned}
$$

Consequently, the matrix model (3.1) is reduced to the Calogero-oscillator model on the $N$-dimensional sphere,

$\mathcal{H}_{\omega}=\frac{\boldsymbol{p}^{2}}{2}-\frac{(\boldsymbol{x} \cdot \boldsymbol{p})^{2}}{2 r_{0}^{2}}+\sum_{i<j} \frac{g^{2}}{\left(x_{i}-x_{j}\right)^{2}}+\frac{\omega^{2} r_{0}^{2}}{2} \frac{x^{2}}{x_{0}^{2}}$.

Thus, the above system can be obtained from the $\mathrm{N}^{2}$ dimensional Higgs oscillator by projecting into the orbits of the adjoint group action (3.12). This projection breaks the Poisson structure: it respects the brackets only among the observables invariant with respect to the reduction group. Therefore, only the $\mathrm{SU}(N)$ invariant constants of motion of the matrix system remain constants in the projected system. They are built by taking an appropriate combination of the elements (3.9), which are preserved under the group action (3.12). In particular, one can construct the following two sets of $\mathrm{SU}(N)$ invariants from the angular momentum and hidden-symmetry generators:

$\mathcal{L}_{2 k}=(\operatorname{tr} \otimes \operatorname{tr}) \mathbf{L}^{2 k}, \quad \mathcal{I}_{k}=(\operatorname{tr} \otimes \operatorname{tr}) \mathbf{I}^{k}, \quad k=1,2,3, \ldots$,

where the left (right) trace from the tensor product tr $\otimes \operatorname{tr}$ is performed over the left (right) factor in the tensor products (3.10) and (3.11). Substituting the explicit expressions obtained from (3.10), (3.11), and (3.15) into the above equations, we arrive at the integrals of motion of the Calogero-oscillator system on the sphere (3.17). Note that the integrals $\mathcal{L}_{2 k+1}$ vanish, since the angular momentum tensor is antisymmetric.

Already the above integrals form an overcomplete set of constants of motion, with enough functionally independent ones to ensure the superintegrability of the system (3.17) discovered in Ref. [9]. Nevertheless, we can construct constants of motion from more general $\mathrm{SU}(N)$ invariants. They are obtained from $L_{a b}$ and $I_{a b}$ using the $\mathrm{SU}(N)$ invariant tensors in the adjoint representation, 


$$
d_{a_{1} \ldots a_{l}}=\operatorname{tr} \mathbf{T}_{a_{1}} \ldots \mathbf{T}_{a_{l}} .
$$

According to (3.3), (3.4) and (3.6),

$d_{a}=\sum_{k=1}^{N} \delta_{k a}, \quad d_{a b}=\delta_{a b}, \quad d_{a b c}-d_{b a c}=\imath f_{a b c}$.

Note that the vector $d_{a}$ projects to the $\mathrm{U}(1)$ generator of the unitary group given by the identity matrix. The higher-rank tensors are more complicated. As a consequence of the completeness condition obeyed by the basis (3.3), they may be expressed in terms of the $d_{a b c}$ by a successive application of the formula

$$
\sum_{c} d_{a_{1} \ldots a_{k} c} d_{c b_{1} \ldots b_{l}}=d_{a_{1} \ldots a_{k} b_{1} \ldots b_{l}} .
$$

Here is an example of a mixed fourth-order (in momenta) invariant:

$$
\sum_{a, a^{\prime}, \ldots} d_{a b c} d_{a^{\prime} b^{\prime} c^{\prime}} L_{a a^{\prime}} L_{b b^{\prime}} I_{c c^{\prime}}
$$

In this context, the integrals (3.18) can be defined alternatively as

$$
\begin{array}{r}
\mathcal{L}_{2 k}=\sum_{a_{1}, b_{1} \ldots, a_{2 k}, b_{2 k}} d_{a_{1} \ldots a_{2 k}} d_{b_{1} \ldots b_{2 k}} L_{a_{1} b_{1}} \ldots L_{a_{2 k} b_{2 k}}, \\
\mathcal{I}_{k}=\sum_{a_{1}, b_{1} \ldots, a_{k}, b_{k}} d_{a_{1} \ldots a_{k}} d_{b_{1} \ldots b_{k}} I_{a_{1} b_{1}} \ldots I_{a_{k} b_{k}} .
\end{array}
$$

By contracting adjacent indices with a Kronecker delta, we arrive at another simple set of invariants,

$$
\begin{array}{r}
\mathcal{L}_{2 k}^{\prime}=\sum_{a_{1} \ldots a_{k}} L_{a_{1} a_{2}} L_{a_{2} a_{3}} \ldots L_{a_{2 k} a_{1}}=\operatorname{Tr} \mathrm{L}^{2 k}, \\
\mathcal{I}_{k}^{\prime}=\sum_{a_{1} \ldots a_{k}} I_{a_{1} a_{2}} I_{a_{2} a_{3}} \ldots I_{a_{k} a_{1}}=\operatorname{Tr} \mathrm{I}^{k} .
\end{array}
$$

Here the rank-four tensors (3.10) and (3.11) are treated as ordinary matrices with entries $I_{a b}$ and $L_{a b}$ correspondingly, i.e. $[\mathrm{I}]_{a b}=I_{a b}$ and $[\mathrm{L}]_{a b}=L_{a b}$. Their counterparts with "free boundaries" are obtained by saturating the first and last indices with the invariant vector $d_{a}$. For example,

$$
\mathcal{I}_{k}^{\prime \prime}=\sum_{i, a_{2} \ldots a_{k}, j} I_{i a_{2}} I_{a_{2} a_{3}} \ldots I_{a_{k} j}=\sum_{i, j=1}^{N}\left(\mathrm{I}^{k}\right)_{i j} .
$$

Let us derive now the explicit form for the second-order (in momentum) integrals. The invariant $\mathcal{L}_{2}$ corresponds to the angular part of the pure Calogero model, whose integrals of motion have been constructed already [17]. It can be presented in terms of the momentum and angular momentum (2.2) as

$$
\mathcal{L}_{2}=2 \sum_{i<j}\left(L_{i j}^{2}+\frac{2 g^{2} x^{2}}{\left(x_{i}-x_{j}\right)^{2}}\right) .
$$

It is equivalent to the $(N-1)$-dimensional Hamiltonian $H_{\Omega}$ (1.4) from the Introduction with the replacement $x \rightarrow r_{0}$ and $x_{N} \rightarrow x_{0}$.

Of course, the constructed integrals are subject to algebraic relations since only $2 N-1$ of them are functionally independent. The first members of the families (3.24) and (3.27) coincide and are equal to

$$
\begin{aligned}
\mathcal{I}_{1} & =\mathcal{I}_{1}^{\prime \prime}=\left(\frac{x_{0} \mathcal{P}}{r_{0}}\right)^{2}+\left(\frac{\omega r_{0} \mathcal{X}}{x_{0}}\right)^{2} \text { with } \\
\mathcal{X} & =\sum_{i=1}^{N} x_{i}, \quad \mathcal{P}=\sum_{i=1}^{N} p_{i} .
\end{aligned}
$$

It is easy to see also that $\mathcal{L}_{2}^{\prime}=-\mathcal{L}_{2}$ and $\mathcal{I}_{2}^{\prime}=\mathcal{I}_{2}$. The Hamiltonian itself is expressed through the constructed integrals as

$$
\mathcal{H}_{\omega}=\frac{1}{2} \mathcal{I}_{1}^{\prime}+\frac{\mathcal{L}_{2}}{4 r_{0}^{2}}
$$

Moreover, the families (3.25), (3.26) and (3.27) do not give rise to new constants of motion. Indeed, the rank-two matrices $L_{a b}$ and $I_{a b}$ are subject to the third-order relations

$$
\mathrm{L}^{3}+\frac{\mathcal{L}_{2}}{2} \mathrm{~L}=0, \quad \mathrm{I}^{3}-\mathcal{I}_{1}^{\prime} \mathrm{I}^{2}+\frac{\omega^{2} \mathcal{L}_{2}}{2} \mathrm{I}=0,
$$

and the surviving first members are already given by (3.29).

In the flat-space limit, $r_{0} \rightarrow \infty$, we obtain the Calogero model with oscillator potential [2]. The generators (3.10) and (3.11) constitute the pure $\mathrm{U}\left(N^{2}\right)$ symmetry of the initial matrix Hamiltonian $\mathcal{H}_{\omega}^{\text {mat }}$. Apart from the Noether integrals (3.13), the other $\mathrm{SU}(N)$ integrals may be expressed in terms of matrix analogs of holomorphic and antiholomorphic variables [1]. In terms of the current phase variables, these quantities are

$$
\begin{aligned}
\mathbf{C} & =\frac{1}{2 \omega} \mathbf{P}^{2}+\frac{\omega}{2} \mathbf{X}^{2}+\frac{1}{2} \mathbf{J}, \\
C_{c} & =\frac{\omega}{2} J_{c}+\frac{1}{2 \omega} \sum_{a, b} d_{a b c} I_{a b}, \\
\left\{C_{a}, C_{b}\right\} & =\sum_{c} f_{a b c} C_{c} .
\end{aligned}
$$

According to our procedure, the constants of motion of the reduced Hamiltonian $\mathcal{H}_{\omega}$ for $r_{0} \rightarrow \infty$, corresponding to the 
aforementioned integrals, can be constructed in the following way:

$$
\mathcal{C}_{k}=\operatorname{tr} \mathbf{C}^{k}=\sum_{a_{1}, \ldots, a_{k}} d_{a_{1} \ldots a_{k}} C_{a_{1}} \ldots C_{a_{k}}
$$

In fact, $\mathcal{C}_{k}$ is a $k$ th-order Casimir element of the $\operatorname{su}(N)$ Poisson algebra for $1 \leq k \leq N$. Therefore, they are in involution, $\left\{\mathcal{C}_{k}, \mathcal{C}_{l}\right\}=0$, and constitute a system of Liouville integrals for the Calogero model in the external oscillator potential [1]. The first element coincides with the Hamiltonian: $\mathcal{H}_{\omega}=\omega \mathcal{C}_{1}$.

\section{B. Calogero-Coulomb on the sphere}

The Hermitian matrix model for the Coulomb system on the sphere is defined by the Hamiltonian

$\mathcal{H}_{\gamma}^{\mathrm{mat}}=\frac{1}{2} \operatorname{tr} \mathbf{P}^{2}-\frac{1}{2 r_{0}^{2}}(\operatorname{tr} \mathbf{P} \mathbf{X})^{2}-\gamma\left(\frac{1}{\operatorname{tr} \mathbf{X}^{2}}-\frac{1}{r_{0}^{2}}\right)^{\frac{1}{2}}$.

In terms of the phase-space variables $\left(P_{a}, X_{a}\right)$, the matrix Hamiltonian (3.34) becomes identical to the Hamiltonian of the spherical Coulomb system (2.11),

$$
H_{\gamma}^{\mathrm{mat}}=\frac{1}{2} \sum_{a} P_{a}^{2}-\frac{1}{2 r_{0}^{2}}\left(\sum_{a} X_{a} P_{a}\right)^{2}-\frac{\gamma}{r_{0}} \frac{x_{0}}{x},
$$

with $x$ and $x_{0}$ defined by (3.8). Apart from the kinematical angular momentum tensor (3.10), it has a conserved Runge-Lenz vector (2.12) given by

$$
A_{a}=\frac{x_{0}}{r_{0}} \sum_{b} L_{a b} P_{b}-\frac{\gamma X_{a}}{x} .
$$

The latter can be presented through the Hamiltonian,

$A_{a}=\left(\frac{2 x_{0}}{r_{0}} \mathcal{H}_{\gamma}^{\mathrm{mat}}+\frac{x_{0}}{r_{0}^{3}}(\boldsymbol{x} \cdot \boldsymbol{p})^{2}-\frac{2 \gamma x}{r_{0}^{2}}+\frac{\gamma}{x}\right) X_{a}-\frac{x_{0}}{r_{0}}(\boldsymbol{x} \cdot \boldsymbol{p}) P_{a}$,

and the corresponding matrix reads

$$
\mathbf{A}=\sum_{a} A_{a} \mathbf{T}_{a}=\frac{x_{0}}{r_{0}}\left(\mathbf{X} \operatorname{tr} \mathbf{P}^{2}-\mathbf{P} \operatorname{tr} \mathbf{X} \mathbf{P}\right)-\frac{\gamma}{x} \mathbf{X} .
$$

The symmetry generators $L_{a b}$ and $A_{a}$ obey the Poisson brackets of the Coulomb system on the sphere; see (2.4) and (2.14).

The $\mathrm{SU}(N)$ reduction procedure implemented above for the Calogero-oscillator system on the sphere remains valid in this case too. The matrix-model Hamiltonian (3.34) can be reduced by the symmetry (3.12) to the CalogeroCoulomb model on the $N$-dimensional sphere,

$$
\mathcal{H}_{\gamma}=\frac{\boldsymbol{p}^{2}}{2}-\frac{(\boldsymbol{x} \cdot \boldsymbol{p})^{2}}{2 r_{0}^{2}}+\sum_{i<j} \frac{g^{2}}{\left(x_{i}-x_{j}\right)^{2}}-\frac{\gamma}{r_{0}} \frac{x_{0}}{x},
$$

where the matrix coordinates $P_{a}$ and $X_{a}$ are defined in (3.16).

The symmetries of the reduced system are described by symmetric polynomials in the reduced angular momentum $L_{a b}$ and Runge-Lenz vector $A_{c}$, with indices coupled by the $\mathrm{SU}(N)$ invariant tensors (3.19) as in the example below,

$$
\sum_{a, a^{\prime}, \ldots} d_{a b c} d_{a^{\prime} b^{\prime} c^{\prime}} L_{a a^{\prime}} L_{b b^{\prime}} A_{c} A_{c^{\prime}}
$$

with $d_{a_{1} \ldots a_{k}}$ defined by (3.19). The invariants depending only on the angular momentum variables agree with $\mathcal{L}_{2 k}$ as defined in (3.23). They are complemented to a full set of integrals by the following simple family of invariants:

$$
\mathcal{A}_{k}=\operatorname{tr} \mathbf{A}^{k}=\sum_{a_{1}, \ldots, a_{k}} d_{a_{1} \ldots a_{k}} A_{a_{1}} \ldots A_{a_{k}} .
$$

Let us write down explicit expressions for the first two integrals from this family. Using the representation (3.37), we immediately get

$\mathcal{A}_{1}=\left(\frac{2 x_{0}}{r_{0}} \mathcal{H}_{\gamma}+\frac{x_{0}}{r_{0}^{3}}(\boldsymbol{x} \cdot \boldsymbol{p})^{2}-\frac{2 \gamma x}{r_{0}^{2}}+\frac{\gamma}{x}\right) \mathcal{X}-\frac{x_{0}}{r_{0}}(\boldsymbol{x} \cdot \boldsymbol{p}) \mathcal{P}$,

where $\mathcal{X}$ and $\mathcal{P}$ are defined in (3.29). As a direct consequence of (2.13), the second integral is expressed via the Hamiltonian (3.39) and the angular Calogero Hamiltonian,

$$
\mathcal{A}_{2}=\left(\mathcal{H}_{\gamma}-\frac{\mathcal{L}_{2}}{4 r_{0}^{2}}\right) \mathcal{L}_{2}+\gamma^{2} .
$$

Thus, using the method of matrix-model reduction, we construct a complete set of constants of motion for the Calogero-oscillator and Calogero-Coulomb models on the $N$-dimensional sphere.

\section{GENERALIZATIONS}

\section{A. Calogero-Coulomb-Stark on the sphere}

Consider an integrable generalization of the CoulombStark system to the sphere [22],

$$
H_{\gamma, F}=H_{\gamma}+\frac{x_{0}}{r_{0}}(\boldsymbol{F} \cdot \boldsymbol{x}),
$$

where $H_{\gamma}$ is given by (2.11) and $\boldsymbol{F}$ is an analog of the constant electric field in the planar limit. This system lacks superintegrability but still remains integrable. Its constants 
of motion are given by the angular-momentum components orthogonal to the electric field direction,

$$
L_{i j}^{\perp}=L_{i j}+n_{i} n_{k} L_{j k}-n_{j} n_{k} L_{i k},
$$

and a modified longitudinal component of the Runge-Lenz vector on the sphere (3.36) given by Ref. [22],

$$
A=\boldsymbol{n} \cdot \boldsymbol{A}-\frac{F}{2}\left(\boldsymbol{x}^{2}-(\boldsymbol{n} \cdot \boldsymbol{x})^{2}\right),
$$

where we introduced the field strength $F=|\boldsymbol{F}|$ and the field direction $\boldsymbol{n}=\boldsymbol{F} / \boldsymbol{F}$.

The matrix-model reduction described in the previous section can be applied to the extension of the system (4.1) by a Calogero potential. The electric field is now characterized by a matrix proportional to the identity, to ensure the $\mathrm{SU}(N)$ invariance of the Stark term under the adjoint action (3.12):

$\mathbf{F}=\frac{F}{\sqrt{N}} \mathbf{1}=\frac{F}{\sqrt{N}} \sum_{k=1}^{N} \mathbf{T}_{k}, \quad \operatorname{tr} \mathbf{F} \mathbf{X}=\frac{F}{\sqrt{N}} \operatorname{tr} \mathbf{X}$.

Therefore, the matrix-model analog of the system on the sphere (4.1) takes the form

$\mathcal{H}_{\gamma, F}^{\text {mat }}=\mathcal{H}_{\gamma}^{\text {mat }}+\frac{F}{\sqrt{N}} \frac{x_{0}}{r_{0}} \operatorname{tr} \mathbf{X}=\mathcal{H}_{\gamma}^{\text {mat }}+\frac{F}{\sqrt{N}} \frac{x_{0}}{r_{0}} \sum_{k=1}^{N} X_{k}$,

with $\mathcal{H}_{\gamma}^{\text {mat }}$ given by (3.34). In other words, the field matrix is tangent to the $\mathrm{U}(1)$ center of the unitary group, and the unit vector along that direction is given by the invariant vector (3.20) as $n_{a}=d_{a} / \sqrt{N}$. So, the conserved transversal components of the angular momentum (4.2) acquire the form

$$
L_{a b}^{\perp}=L_{a b}+\frac{1}{N} \sum_{k=1}^{N}\left(d_{a} L_{b k}-d_{b} L_{a k}\right),
$$

where the first equation in (3.20) has been used.

The modified component of the Runge-Lenz vector tangent to the $\mathrm{U}(1)$ center is given by

$$
A=\frac{1}{\sqrt{N}} \operatorname{tr} \mathbf{A}-\frac{F}{2}\left(\operatorname{tr} \mathbf{X}^{2}-\frac{1}{N}(\operatorname{tr} \mathbf{X})^{2}\right) .
$$

Reducing the matrix model (4.5) by the $\mathrm{SU}(N)$ group action, we arrive at the Calogero-Coulomb-Stark Hamiltonian on the sphere:

$$
\mathcal{H}_{\gamma, F}=\mathcal{H}_{\gamma}+\frac{F}{\sqrt{N}} \frac{x_{0}}{r_{0}} \mathcal{X}
$$

with $\mathcal{X}$ given in (3.29).

Projecting the matrix-model integrals (4.6) and (4.7) to the $\mathrm{SU}(N)$ invariant orbits in the usual way, we arrive at the following constants of motion for the above Hamiltonian:

$$
\begin{gathered}
\mathcal{L}_{2 k}^{\perp}=\sum_{a_{1}, b_{1} \ldots, a_{2 k}, b_{2 k}} d_{a_{1} \ldots a_{2 k}} d_{b_{1} \ldots b_{2 k}} L_{a_{1} b_{1}}^{\perp} \ldots L_{a_{2 k} b_{2 k}}^{\perp}, \\
\mathcal{A}=\frac{1}{\sqrt{N}} \mathcal{A}_{1}-\frac{F}{2}\left(\boldsymbol{x}^{2}-\frac{\mathcal{X}^{2}}{N}\right),
\end{gathered}
$$

where the definition (3.42) is taken into account. The above constants of motion ensure the integrability of the system.

In the $r_{0} \rightarrow \infty$ limit, the Hamiltonian (4.8) describes the Calogero-Coulomb-Stark problem in flat space, which has been introduced and studied in Ref. [12] using the Dunkl operator approach.

\section{B. Spin extensions}

The aforementioned systems on the sphere can be endowed with an additional classical spin while retaining their integrability or superintegrability. Such extensions have been studied in the flat-space limit for the Calogero model using the Lax pair technique, by introducing internal degrees of freedom $l_{i j}$ into the inverse-square potential of the system (3.17) [23]. In particular, the Calogero-oscillator Hamiltonian on the sphere (3.1) with classical spins has the following form:

$\mathcal{H}_{\omega}^{\text {spin }}=\frac{\boldsymbol{p}^{2}}{2}-\frac{(\boldsymbol{x} \cdot \boldsymbol{p})^{2}}{2 r_{0}^{2}}+\sum_{i<j} \frac{l_{i j}^{2}}{\left(x_{i}-x_{j}\right)^{2}}+\frac{\omega^{2} r_{0}^{2}}{2} \frac{x^{2}}{x_{0}^{2}}$

The spin dynamic variables $l_{i j}$ obey $s o(N)$ angular momentum Poisson bracket relations (2.4), but they are in involution with the variables $p_{i}$ and $x_{i}$ describing the motion on the sphere. They can be recast into a spin degree of freedom $S$ related to each coordinate: $l_{i j}=S_{i} \cdot S_{j}$ [24].

Actually, the classical spin $l_{i j}$ can be obtained also from the matrix-model reduction procedure $[1,8,24]$. Remember that the Noether generators (3.13) form a traceless Hermitian matrix, and so far we took the minimal gauge (3.14) for them. Let us instead apply a spin gauge by imposing a weaker condition: $J_{i j}=\imath l_{i j}$. Using the antisymmetry of the $l_{i j}$, the conventional Lax matrix (3.15) gets replaced by

$$
P_{i j}=p_{i} \delta_{i j}-\left(1-\delta_{i j}\right) \frac{l_{i j}}{x_{i}-x_{j}} .
$$

The construction scheme for the integrals of motion described in Sec. III can be extended to the spin case too. 
The integrals are given by the $\mathrm{SU}(N)$ invariant polynomials built from the spin matrix $l_{i j}$ and the reduced matrices $X_{i j}$ and $P_{i j}$. In the flat-space limit, the algebra of integrals of the Calogero system with and without oscillator terms has been studied in this context in Ref. [25].

\section{CONCLUDING REMARKS}

We have defined the classical rational Calogero model with an oscillator or Coulomb potential on the sphere or hyperboloid. This may be viewed as the oscillator or Coulomb system on the sphere or hyperboloid, as introduced, respectively, by Higgs and Schrödinger [13,14], amended by a Calogero interaction term. This system looks similar to, but is distinct from, the angular part of the Calogero model in the ambient $(N+1)$-dimensional flat space. Both systems, however, share features such as superintegrability, a Lax pair, and matrix-model descriptions.

We have focused on the constants of motion for the Calogero-oscillator and Calogero-Coulomb systems on the sphere. They were obtained from the kinematical and hidden dynamical symmetries of the related Hermitian matrix models by a Hamiltonian reduction. We have expressed the constants of motion of the reduced systems as $\mathrm{SU}(N)$ invariant polynomials depending on the wellknown integrals of the original matrix model. We also have studied the spherical generalization of the CalogeroCoulomb system in an external electric field, i.e. with a Stark term, and briefly discussed the effect of additional spin degrees of freedom.

In a forthcoming paper, we will extend the construction carried out here to the quantum case [26]. It will be interesting to consider in this context the spherical Calogero models associated with general Coxeter root systems.

\section{ACKNOWLEDGMENTS}

T. H. and A.N. were partially supported by the Armenian State Committee of Science Grants No. 15RF039 and No. 15T-1C367, and by Grant No. mathph-4220 of the Armenian National Science and Education Fund based in New York (ANSEF). The work of T. H. and A. N. was done within the ICTP programs NET68 and OEA-AC-100 and within the program of Regional Training Networks on Theoretical Physics by VolkswagenStiftung Contract No. 86 260. The work of F. C. is supported by the Alexander von Humboldt Foundation under Grant No. CHL 1153844 STP.
[1] A. P. Polychronakos, Physics and mathematics of Calogero particles, J. Phys. A 39, 12793 (2006).

[2] F. Calogero, Solution of a three-body problem in onedimension, J. Math. Phys. (N.Y.) 10, 2191 (1969); Solution of the one-dimensional $N$-body problems with quadratic and/or inversely quadratic pair potentials, J. Math. Phys. (N.Y.) 12, 419 (1971).

[3] J. Moser, Three integrable Hamiltonian systems connected with isospectral deformations, Adv. Math. 16, 197 (1975).

[4] M. A. Olshanetsky and A. M. Perelomov, Classical integrable finite dimensional systems related to Lie algebras, Phys. Rep. 71, 313 (1981); Quantum integrable systems related to Lie algebras, Phys. Rep. 94, 313 (1983).

[5] S. Wojciechowski, Superintegrability of the CalogeroMoser system, Phys. Lett. A 95, 279 (1983).

[6] C. Gonera, On the superintegrability of Calogero-MoserSutherland model, J. Phys. A 31, 4465 (1998).

[7] B. Sutherland, Exact results for a quantum many-body problem in one dimension, Phys. Rev. A 4, 2019 (1971); Exact results for a quantum many-body problem in one dimension: II, Phys. Rev. A 5, 1372 (1972).

[8] A. Polychronakos, Multidimensional Calogero systems from matrix models, Phys. Lett. B 408, 117 (1997).

[9] T. Hakobyan, O. Lechtenfeld, and A. Nersessian, Superintegrability of generalized Calogero models with oscillator or Coulomb potential, Phys. Rev. D 90, 101701(R) (2014).
[10] T. Hakobyan and A. Nersessian, Runge-Lenz vector in Calogero-Coulomb problem, Phys. Rev. A 92, 022111 (2015).

[11] M. Adler, Some finite dimensional integrable systems and their scattering behavior, Commun. Math. Phys. 55, 195 (1977); C. Gonera and P. Kosinski, Calogero model and $s l(2, R)$ algebra, Acta Phys. Pol. B 30, 907 (1999).

[12] T. Hakobyan and A. Nersessian, Integrability and separation of variables in Calogero-Coulomb-Stark and two-center Calogero-Coulomb systems, Phys. Rev. D 93, 045025 (2016).

[13] E. Schrödinger, A method of determining quantummechanical eigenvalues and eigenfunctions, Proc. R. Irish Acad., Sect. A 46, 9 (1940).

[14] P. W. Higgs, Dynamical symmetries in a spherical geometry: I, J. Phys. A 12, 309 (1979).

[15] A. Galajinsky, A. Nersessian, and A. Saghatelian, Superintegrable models related to near horizon extremal MyersPerry black hole in arbitrary dimension, J. High Energy Phys. 06 (2013) 002.

[16] T. Hakobyan, D. Karakhanyan, and O. Lechtenfeld, The structure of invariants in conformal mechanics, Nucl. Phys. B886, 399 (2014); T. Hakobyan, S. Krivonos, O. Lechtenfeld, and A. Nersessian, Hidden symmetries of integrable conformal mechanical systems, Phys. Lett. A 374, 801 (2010); T. Hakobyan, A. Nersessian, and V. Yeghikyan, The cuboctahedric Higgs oscillator from 
the rational Calogero model, J. Phys. A 42, 205206 (2009).

[17] T. Hakobyan, O. Lechtenfeld, and A. Nersessian, The spherical sector of the Calogero model as a reduced matrix model, Nucl. Phys. B858, 250 (2012).

[18] M. Feigin, O. Lechtenfeld, and A. Polychronakos, The quantum angular Calogero-Moser model, J. High Energy Phys. 07 (2013) 162; F. Correa and O. Lechtenfeld, The tetrahexahedric angular Calogero model, J. High Energy Phys. 10 (2015) 191.

[19] M. Feigin and T. Hakobyan, On Dunkl angular momenta algebra, J. High Energy Phys. 11 (2015) 107.

[20] D. M. Fradkin, Existence of the dynamic symmetries $O_{4}$ and $\mathrm{SU}_{3}$ for all classical central potential problems, Prog. Theor. Phys. 37, 798 (1967); Three-dimensional isotropic harmonic oscillator and $S_{3}$, Am. J. Phys. 33, 207 (1965).

[21] D. Kazhdan, B. Kostant, and S. Sternberg, Hamiltonian group actions and dynamical systems of Calogero type, Commun. Pure Appl. Math. 31, 481 (1978).

[22] A. Nersessian and V. Yeghikyan, Anisotropic inharmonic Higgs oscillator and related (MICZ-) Kepler-like systems, J. Phys. A 41, 155203 (2008); Anisotropic Higgs oscillator, in Proceedings of VIIth International Workshop on Supersymmetries and Quantum Symmetries (SQS'07), Dubna, Russia, 2007, arXiv:0711.1033; S. Bellucci and V. Yeghikyan, The Coulomb problem on a 3-sphere and Heun polynomials, J. Math. Phys. (N.Y.) 54, 082103 (2013).

[23] S. Wojciechowski, An integrable marriage of the Euler equations with the Calogero-Moser system, Phys. Lett. A 111, 101 (1985); J. Gibbons and T. Hermsen, A generalisation of the Calogero-Moser system, Physica D (Amsterdam) 11, 337 (1984).

[24] J. A. Minahan and A. P. Polychronakos, Interacting fermion systems from two dimensional QCD, Phys. Lett. B 326, 288 (1994).

[25] J. Avan and E. Billey, Observable algebras for the rational and trigonometric Euler-Calogero-Moser models, Phys. Lett. A 198, 183 (1995); J. Avan, Integrable extensions of the rational and trigonometric $A_{N}$ Calogero-Moser potentials, Phys. Lett. A 185, 293 (1994).

[26] F. Correa, T. Hakobyan, O. Lechtenfeld, and A. Nersessian, Spherical Calogero model with oscillator/Coulomb potential: Quantum case, Phys. Rev. D 93, 125009 (2016). 